\title{
Malnutrition in patients with chronic kidney disease
}

\author{
Sungjin Chung, Eun Sil Koh, Seok Joon Shin, Cheol Whee Park* \\ Division of Nephrology, Department of Internal Medicine, College of Medicine, The Catholic University of Korea, Seoul, Republic \\ of Korea \\ Email: * cheolwhee@hanmail.net
}

Received 19 January 2012; revised 9 March 2012; accepted 5 April 2012

\begin{abstract}
Malnutrition is a major issue in patients with chronic kidney disease (CKD), adversely affecting morbidity, mortality, functional activity and patients' quality of life. Our knowledge of the pathogenic mechanisms of malnutrition in patients with CKD, including endstage renal disease, has been improved. This has led to the development of clinical practice guidelines for nutritional care in CKD which provide a framework for the nutritional issues facing patients and physiccians. Extensive research in the field of nutrition in patients with CKD has resulted in the formation of general guidelines, although some uncertainties still exist on some of the best therapeutic or preventive options in uremic malnutrition. It is important to search actively for malnutrition since early diagnosis and treatment can improve the prognosis for CKD patients and reduce the monetary costs connected with treatment.
\end{abstract}

Keywords: Chronic Kidney Disease; End-Stage Renal Disease; Inflammation; Malnutrition

\section{INTRODUCTION}

Chronic kidney disease (CKD), characterized by progressive deterioration of renal function, is a growing problem which has potential public health consequences. The total number of CKD patients has markedly increased during the last 30 years [1,2], and the prevalence of CKD has reached epidemic proportions with $10 \%$ $13 \%$ of the populations of Korea, Taiwan, Iran, Japan, China, Canada, India and the USA being affected. This number will undoubtedly rise in coming years if the prevalence of diabetes and hypertension continue to increase [3]. Furthermore, the increase in the elderly population and the wider availability of therapy such as dialysis and kidney transplantation, contribute to the increasing prevalence of patients with CKD [4]. The

*Corresponding author. socio-economic impact of CKD and its complications are also considerable [2]. Although CKD patients account for $9.8 \%$ of the total Medicare population in the USA, these patients account for as much as $27.6 \%$ of total Medicare costs [2]. It has been estimated that the total worldwide cost for treating patients with CKD exceeds one trillion US dollars. CKD is clinically divided into 5 stages based upon the GFR and signs of kidney damage [5]. Stage 5 CKD, kidney failure or end-stage renal disease (ESRD), represents the total failure of the kidneys to maintain metabolic homeostasis, and this state is incompatible with life. A study in the United Kingdom demonstrated that the prevalence of stage 3 - 5 CKD was $8.5 \%$ [2]. Since 26 studies found a prevalence of CKD of $7.2 \%$ in patients older than 30 years, and a prevalence from $23.4 \%$ to $35.8 \%$ in those older than 64 years, CKD should be considered a public health priority [6].

Malnutrition is usually defined as poor nutritional status resulting from poor nutrient intake. However, complex factors other than inadequate intake are the main cause of the nutritional and metabolic derangements in uremic patients. In these patients, serum and tissue proteins tend to be low despite dietary protein and energy intake that is based on standard nutritional guidelines [7]. In addition, some CKD patients have low levels of protein stores regardless of their weight, with some actually being overweight. Although there is no totally adequate definition for such a status in CKD patients, "uremic malnutrition" is the commonly used term. [7]. Because of the many different diagnostic tools used in unrelated studies, the prevalence of malnutrition varies widely among different reports, ranging from $20 \%$ - $50 \%$ at different stages of CKD $[7,8]$.

The kidneys are essential for maintaining many aspects of the metabolic homeostasis [9]. The main functions of the kidneys include elimination of waste products, regulation of water, electrolyte and acid-base balance, and the synthesis and regulation of hormones. In addition, the kidney is one of the principal organs involved in nutritional balance in the body. For the regulation of glucose metabolism, the kidney exhibits glucose synthetic rates several times higher than that 
observed in the liver [10]. Release of glucose by the kidney is known to account on average for up to $20 \%$ of all glucose released after primary absorption into the circulation [11-14]. Because the kidney normally stores little glycogen and renal cells that could store glycogen lack glucose-6-phosphatase, glucose release from the kidney is thought to be predominantly due to gluconeogenesis [14]. Renal glucose release is of the same order of magnitude as splanchnic glucose release during the postabsorptive period in the body. It appears to be more sensitive to hormonal action than hepatic glucose release, and may have a more important role during adaptation to various physiologic and pathologic conditions. Although renal gluconeogenesis has different substrate requirements and demands various gluconeogenic precursors such as lactate, glutamine, glycerol, $\alpha$-ketoglutarate and citrate, lactate is the most predominant and important precursor for renal gluconeogensis as well as systemic gluconeogenesis in humans. According to animal and human studies, only the proximal tubule is capable of synthesizing glucose, and is the only nephron segment that contains the key gluconeogenic enzymes glucose 6-phosphatase, fructose 1,5-diphosphatase, and phosphoenolpyruvate carboxykinase. Gluconeogenic ability in the proximal tubule has a reciprocal relationship with solute transport in the kidney. Therefore, the renal contribution to systemic gluconeogenesis might change under different physiologic or pathophysiologic states such as hypoglycemia, postprandial status, acidbase disorders, and diabetes.

The human kidney also plays a major role in the homeostasis of body amino acid pools, which is carried out by the synthesis, degradation, filtration, reabsorption and excretion of amino acids in the renal tubules. Approximately 50 - 70 g per day of amino acids are filtered by normally functioning kidneys and they are almost completely reabsorbed by the proximal tubules [15]. In addition, the kidneys can do fine tune the circulating and tissue pools of amino acids in the body. They are involved in the main disposal of glutamine and proline from the blood and the net release of some amino acids such as serine, tyrosine and arginine. The kidneys also release smaller amounts of threonine, lysine and leucine into the systemic circulation. Therefore, it is natural that progressive alterations in renal function or metabolism can cause progressive effects on nutrition, as well as on cardiovascular status [15].

Once CKD develops, many kinds of metabolic changes due to kidney disease, underlying co-morbidities and dialysis procedure occur. Many factors lead to serious nutritional complications for CKD patients during the course of predialysis and dialysis, which eventually affect the prognosis and quality of life of patients with CKD (Figure 1).

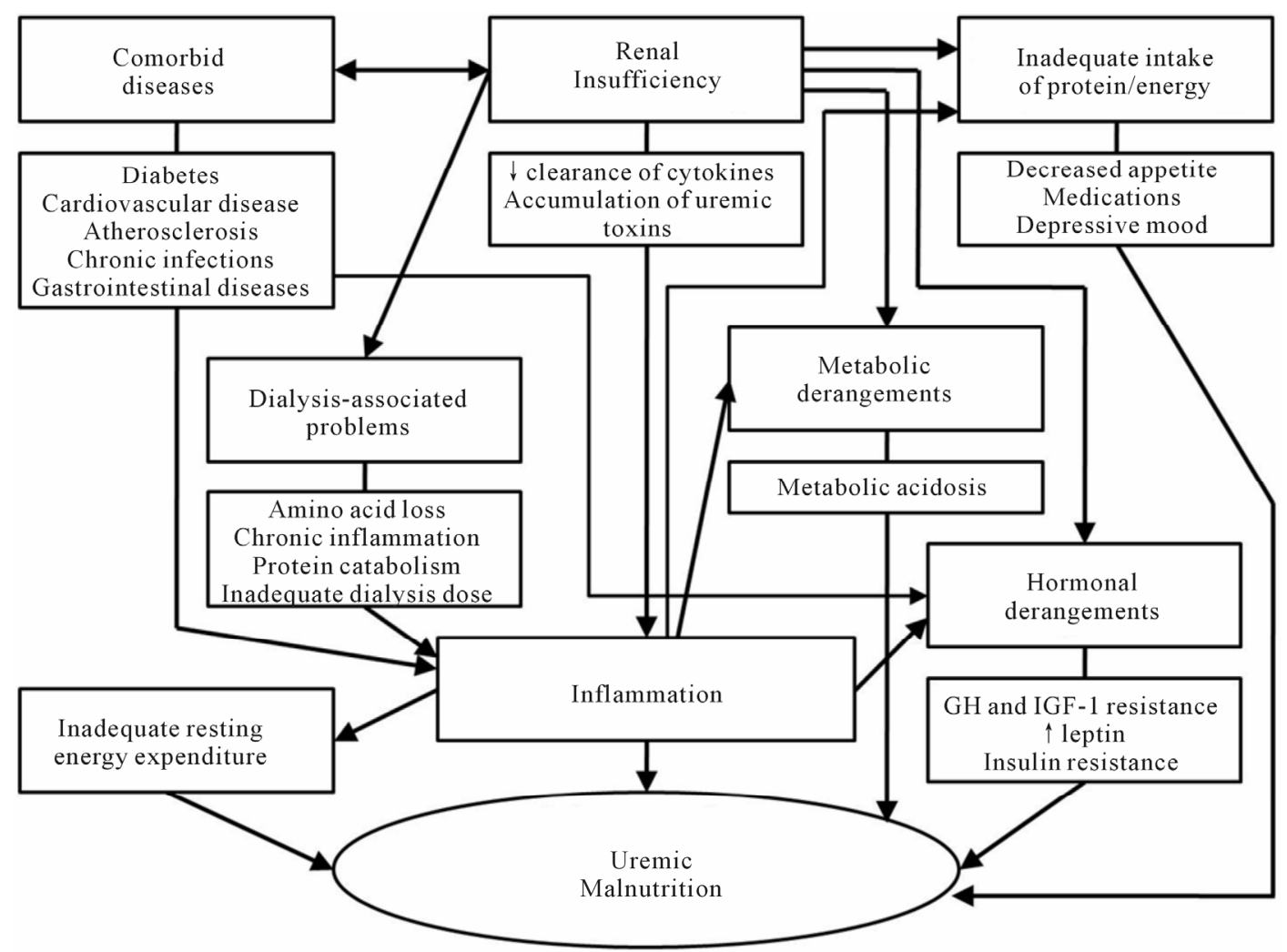

Figure 1. Etiologic factors for malnutrition in chronic kidney disease. 


\section{CHRONIC KIDNEY DISEASE AND MALNUTRITION}

\subsection{Disorders of Protein Metabolism}

Almost all CKD patients, including diet-induced obesityrelated CKD, are gradually affected by low protein and energy intake due to restrictive dietary prescriptions, poor appetite, and uremia-related anorexia. This "protein energy malnutrition" or "protein energy wasting", classically known as poor nutritional status due to inadequate nutrient intake, is a major problem because proper nutrients are the substrate necessary for cell and tissue development and homeostasis. A strict low-protein diet may have a negative effect on nitrogen balance in the predialysis period [4]. A safe low-protein diet should contain a minimum $0.6 \mathrm{~g}$ of protein $/ \mathrm{kg}$ per day.

Protein energy malnutrition due to inadequate nutrient intake alone is relatively uncommon even in CKD. Several studies have reported that healthy subjects, as well as CKD patients who have not reached ESRD, are usually able to maintain neutral nitrogen balance with lower levels of protein intake if energy intake is adequate.

This compensatory response appears to be mediated through a combination of metabolic adjustments, such as a reduction in amino acid oxidation, and protein breakdown and an increase in protein synthesis, all in an attempt to maintain neutral nitrogen balance [16]. Therefore, derangements in protein metabolism leading to malnutrition is observed more often in an advanced CKD or ESRD patients [4,15]. Uremic malnutrition is present in approximately $20 \%$ - $50 \%$ of dialysis patients and is characterized by the insidious loss of somatic protein stores, reflected in lean body mass. In this case, serum concentration abnormalities of visceral proteins, such as serum albumin, prealbumin, transferrin, and cholesterol are developed [17]. Urinary protein loss and removal of amino acids during a dialysis may also play a role. In addition, metabolic acidosis contributes to negative nitrogen and total body protein balance in CKD patients $[18,19]$.

On the other hand, decreased energy availability in CKD patients appears to be responsible for a reduced protein synthesis rates. Muscle biopsies obtained in CKD patients showed significantly lower synthetic rates of myosin heavy chain, mitochondrial protein, muscle cytochrome c-oxidase activity and citrate synthase [20]. Reduced bioavailability of insulin-like growth factor (IGF)-1 may also play a role in decreasing muscle protein synthesis, which is due to increased serum binding proteins. Growth hormone (GH)-induced changes in muscle protein synthesis are related to changes in insulin-like growth factor binding protein (IGFBP)-1 and in the IGF-1/IGFBP-3 ratio. As circulating IGFBP-1 and IGFBP-2 increase in the blood of CKD patients, owing to the diminished renal clearance and the inflammatory status, changes in muscle protein synthesis dependent on the availability of free IGF-1 levels occur [20].

\subsection{Disorders of Carbohydrate and Lipid Metabolism}

Disorders of carbohydrate metabolism are frequent in CKD. As noted above, insulin resistance is primarily detectable when the GFR is less than $50 \mathrm{ml} / \mathrm{min}$. Nondiabetic CKD patients also have glucose intolerance, probably because of peripheral insulin resistance [21]. Besides insulin resistance, reduced insulin-mediated non-oxidative glucose disposal is the most evident defect of glucose metabolism, but impairments of glucose oxidation, the defective suppression of endogenous glucose production, and abnormal insulin secretion also contribute to uremic glucose intolerance [22]. Reduced insulin-mediated non-oxidative glucose disposal is the most evident defect of glucose metabolism in CKD patients. As noted above, insulin resistance is primarily detectable when the GFR is less than $50 \mathrm{ml} / \mathrm{min}$. Nondiabetic as well as diabetic CKD patients have glucose intolerance, probably because of peripheral insulin resistance [21]. Accumulation of nitrogenous uremic toxins seems to be the dominant cause of the specific defect in insulin action, and identification of these toxins is progressing, particularly in the field of carbamylated amino acids. In addition, impairments of glucose oxidation, the defective suppression of endogenous glucose production, and abnormal insulin secretion also contribute to uremic glucose intolerance [22]. Accumulation of nitrogenous uremic toxins seems to be the dominant cause of the specific defect in insulin action, and identification of these toxins is progressing, particularly in the field of carbamylated amino acids. The consequences of ESRD, such as exercise intolerance, anemia, metabolic acidosis, secondary hyperparathyroidism, or vitamin D deficiency, also indirectly play a role in glucose derangements of CKD patients as well $[22,23]$.

Serum triglycerides are elevated in ESRD patients because of enhanced production of triglyceride-rich lipoproteins such as very-low-density lipoproteins in the liver [24] and because of dysfunction of triglyceride degradation resulting from insufficient mitochondrial beta-oxidation of fatty acids. It can be caused by a deficit of L-carnitine, which absence is frequently found, especially in hemodialysis (HD) patients [4,25]. Hyperinsulinemia is the main factor increasing the synthesis of triglycerides and also directly decreasing the activity of lipoprotein lipase. The most prominent changes in lipid metabolism found in many patients with ESRD are increased serum triglyceride levels and low levels of high-density lipoprotein (HDL) cholesterol. Low-density 
lipoprotein (LDL) cholesterol levels are often normal, but the cholesterol may originate from the atherogenic small and dense LDL subclass. The apolipoprotein Bcontaining part of the lipoprotein may undergo modifications such as peptide modification of enzymatic and advanced glycation end-products, oxidation or glycation. Modifications contribute to impaired LDL receptor-mediated clearance from the plasma and promote prolonged circulation of LDL, and HDL particles become structurally altered due to inflammation. Despite conflicting reports, hypercholesterolemia, obesity, and increased blood levels of creatinine and homocysteine paradoxically appear to be associated with a more favorable outcome in ESRD patients [26,27].

The most prominent changes in lipid metabolism found in ESRD patients are increased serum triglyceride levels and low levels of HDL cholesterol. Serum triglycerides elevation is due to enhanced production of triglyceride-rich lipoproteins such as very-low-density lipoproteins in the liver [24]. Furthermore, dysfunction of triglyceride degradation resulting from insufficient mitochondrial beta-oxidation of fatty acids occurred, which can be caused by a deficit of L-carnitine, especially in HD patients $[4,25]$. Hyperinsulinemia is one of the main factors increasing the synthesis of triglycerides, and it also directly decreases the activity of lipoprotein lipase. The apolipoprotein B-containing part of the lipoprotein may undergo modifications including peptide modification of enzymatic and advanced glycation end-products, oxidation or glycation. These modifications contribute to impaired LDL receptor-mediated clearance from the plasma and promote prolonged circulation of LDL, and HDL particles become structurally altered due to inflammation [27].

\subsection{Chronic Inflammation}

In patients with CKD, the most important factor associated with uremic malnutrition is "inflammation", which is a complex combination of physiologic, immunologic, and metabolic effects occurring in response to a diversity of internal or external stimulants. Many cytokines including interleukin (IL)-1, IL-6, and tumor necrosis factor (TNF)- $\alpha$ seem to be primarily involved in the inflammatory process in CKD and ESRD patients. Considering that several pro-inflammatory cytokines are removed by the normal kidney, the decrease of renal IL-6 clearance could, at least in part, account for the increase in IL-6 levels in patients with CKD and ESRD patients [28]. IL-6 levels increase in blood when the glomerular filtration rate progressively declines, which suggests that the reduced renal metabolic activity might be responsible for the increase in cytokines. Clearly, pro-inflammatory cytokines are associated with the development of ano- rexia and/or suppression of nutrient intake. According to experimental studies, cytokines appear to exert direct actions on the satiety center, and further, TNF- $\alpha$ administration causes increased skeletal muscle protein breakdown [29]. IL-6 is also associated with increased muscle proteolysis, and the administration of IL-6 receptor antibody can block this effect [29]. Leptin, a member of the IL-6 superfamily of cytokines, is produced in adipocytes and its primary site of action is on the brain to inhibit the satiety center [30]. The fractional extraction of leptin by the kidney appears to vary little between $9 \%$ to $13 \%$ and leptin may be removed by the splanchnic organs in lean subjects [31]. Increased levels of leptin in dialysis patients are associated with markers of poor nutritional status, suggesting that hyperleptinemia may be another causative factor of cachexia. Considering that the main metabolic effects of these cytokines are catabolic [32], it is critical to suppress the activities of cytokines, at least in theory. Unfortunately, the existence of the ongoing inflammatory response and irreversible comorbid diseases make control of the cytokines hardly feasible. Besides, the relative contributions of protein energy wasting and inflammation to mortality remain obscured because many indicators of inflammation and wasting coexist [33].

Chronic inflammation also induces cytokine-mediated hypermetabolism. Increased resting energy expenditure (REE), which is one of three major components of total daily energy expenditure (TEE), is observed in most chronic inflammatory conditions such as congestive heart failure, rheumatoid arthritis, and various cancers $[34,35]$. Although the exact mechanism of the increased REE in patients with chronic inflammatory disease is not clear, a high REE has been observed in association with increased concentrations of pro-inflammatory cytokines, and ESRD patients also have increased REE [36]. Other indirect effects of chronic inflammation predisposing CKD patients to hypercatabolism include a decrease in voluntary activity and the presence of an underlying disease requiring bed rest [37]. A prolonged decrease in muscle activity is associated with muscle weakness, muscle atrophy, and negative nitrogen balance, finally leading to loss of lean body mass.

\subsection{Oxidative Stress}

In association with uremia and chronic inflammation, oxidative stress may threaten CKD and ESRD patients with serious metabolic complications. Oxidative stress is the state in which the production of reactive oxygen species (ROS) exceeds the capacity of the antioxidant defense system in cells and tissues [4]. ROS act as free radicals, highly reactive substances with an unpaired electron in the outer orbit, and other related reactive 
compounds such as hydrogen peroxide and hypochlorous acid [38]. Free radicals can be produced from leukocytes activated by contacting with the dialysis membrane, and from erythrocyte iron released due to hemolysis [4]. Intravenous administration of iron can also contribute to oxidative stress [39], and co-administrated ascorbic acid, given with the goal of mobilizing iron stores, can further stimulate free radical formation, possibly by reduction of Fe (III) ions to more oxidative Fe (II) compounds [4]. ROS and its related compounds can attack lipids, proteins and nucleic acids and alter the structure and function of these macromolecules [4,38]. LDL particles are especially damaged by excessive oxidation and consequently are not recognized by cell LDL receptors. These damaged LDL particles subsequently accumulate in the blood, leading to nutritional derangements.

\subsection{Hormonal Derangements}

The chronic inflammation in CKD patients decreases anabolism such as protein synthesis, fat mobilization and gluconeogenesis. In addition, metabolic acidosis impairs the action of several anabolic hormones, including GH, thyroid hormone and insulin [40-42]. GH exerts several anabolic actions, and IGF-1 is one of the major mediators of these actions in adults. Hormonal activities by compounds of GH or IGF-1 are disrupted in CKD patients. Uremia is known to be associated with reduced expression of hepatic $\mathrm{GH}$ receptor mRNA and hepatic IGF-1 mRNA, as well as a defect in GH signal transduction [40,41]. The abnormalities of this hormonal axis constitute an important factor in the pathogenesis of uremic malnutrition. Uremia is also characterized by insulin resistance. Insulin binding to its receptor seems to be normal in CKD patients, however, a post-receptor defect in tissue insulin responsiveness is observed, resulting in insulin resistance. Decreased food intake can also contribute to insulin resistance.

\subsection{Metabolic Acidosis}

Metabolic acidosis is noted in a majority of patients when the GFR decreases to less than 20\% - 25\% of normal [4], and the degree of acidosis correlates with the severity of CKD. Several adverse consequences are associated with uremic acidosis, including muscle wasting, mineral-bone disease, impaired insulin sensitivity and exacerbation of beta2-microglobulin accumulation [43]. Metabolic acidosis causes an alteration of protein balance, which is shown by an increase in leucine oxidation and/or protein degradation. Inverse correlations between net protein balance and blood bicarbonate are also observed, suggesting that acidosis is responsible for the more negative protein balance [44-46]. Other complications associated with metabolic acidosis in
CKD include anorexia, fatigue, impaired function of the cardiovascular system, hyperkalemia, and altered gluconeogenesis and triglyceride metabolism [18].

\subsection{Dialysis-Related Problems}

There is a slight difference between CKD not requiring immediate dialysis and ESRD patients maintained on dialysis. Once CKD progress to ESRD and patients initiate maintenance dialysis, their dietary protein intake increases, at least during the first year of therapy [26,47]. Despite increase in dietary protein and energy intake after initiation of dialysis, the high prevalence of a poor nutritional status is developed, which suggests that protein energy malnutrition alone does not explain the poor nutritional status in dialysis patients. The aggravated nutritional status in dialysis patients seems to be associated with the dialysis procedure, as well as with more advanced uremia. During standard HD treatment using high-flux dialyzers, approximately $8 \mathrm{~g}$ of free amino acids are removed [48]. In addition, HD is known as a catabolic procedure, which is evidenced by the fact that patients on dialysis days are in negative nitrogen balance, even with high levels of protein intake [37]. A study using stable isotope tracer technique reported that the catabolic effects of HD were limited to amino acid losses. Furthermore, it was reported that increased net whole-body and muscle protein loss persisted for at least 2 hours after completion of an HD treatment [49].

HD membrane can activate the complement system and contribute to the inflammatory process in HD patients. The use of biocompatible membrane during HD procedure has been recommended because of the beneficial nutritional effects, such as higher concentrations of serum albumin and serum IGF-1, and higher weight gain compared to the use of bioincompatible membranes [50]. However, these derangements have also been observed with the use of biocompatible HD membranes and relatively pure dialysate, suggesting that the HD procedure itself initiates certain metabolic pathways leading to both decreased protein synthesis and increased protein breakdown [37,51]. In addition, REE in ESRD patients is further increased by HD, even using biocompatible membranes [52]. Following HD, diminished carbohydrate and accelerated lipid and amino acid oxidation is also observed.

\section{ASSESSMENT OF MALNUTRITION IN PATIENTS WITH CHRONIC KIDNEY DISEASE}

Prior to embarking on nutritional intervention, it is important to grasp the nutritional status in CKD and ESRD patients. A variety of tools and techniques available to assess nutritional status in CKD patients are 
shown in Table 1. An ideal and reliable nutritional marker should either predict clinically important outcomes or identify patients who should receive nutritional management. For many years, nephrologists have been concerned about the validity of the biologic markers used to evaluate nutritional status in CKD and ESRD patients. The most commonly used clinical marker is serum albumin. A large number of studies have shown that serum albumin is a reliable indicator of nutritional status, and that it also displays a notable response to nutritional intervention [53,54]. Differing from normal subjects, CKD and ESRD patients have substantially altered total body water distribution and experience frequent changes in plasma volume, both of which are known to affect albumin turnover and consequently serum albumin concentrations $[55,56]$. Furthermore, a chronic inflammatory condition in CKD and ESRD patients is known to influence albumin turnover [51,55,56]. Despite inherent limitations, serum albumin is routinely assessed to identify potentially low protein stores and nutritional status in CKD and ESRD patients.

Serum pre-albumin and transferrin can be alternatives to serum albumin as nutritional markers in CKD and ESRD patients. These markers appear to have certain advantages in that they provide an earlier response to nutritional changes and they can be more precisely measured. However, both serum pre-albumin and serum transferrin are also affected by inflammatory conditions. Pre-albumin is excreted through the kidney and transferrin is closely related to iron metabolism. Neither of these has been studied as thoroughly as albumin in CKD and ESRD patients [57-59] and further, it is expensive in some countries to check them regularly.

Plasma homocysteine concentration may reflect nutritional status in ESRD [60,61]. Although hyperhomocysteinemia is present in the majority of CKD and ESRD patients, plasma levels of homocysteine are higher in CKD patients with appropriate nutritional status than in malnourished patients. In addition, the plasma homocysteine level is inversely correlated with SGA and positively correlated with serum albumin and protein intake.

Measurement of somatic protein stores can be used for assessment of nutritional status in ESRD patients. Anthropometrics, lean body mass measurements by dualenergy X-ray absorptiometry (DEXA) or total body nitrogen (TBN) have been studied in this population [51]. DEXA appears to be the most reliable body composition method for evaluating the ESRD population. It relies on fewer assumptions regarding the influence of fluid status on fat mass measurements compared to bioelectrical impedance analysis (BIA) [62].

BIA has been reported known as an accurate method

Table 1. A variety of tools and techniques available to assess nutritional status in patients with chronic kidney disease.

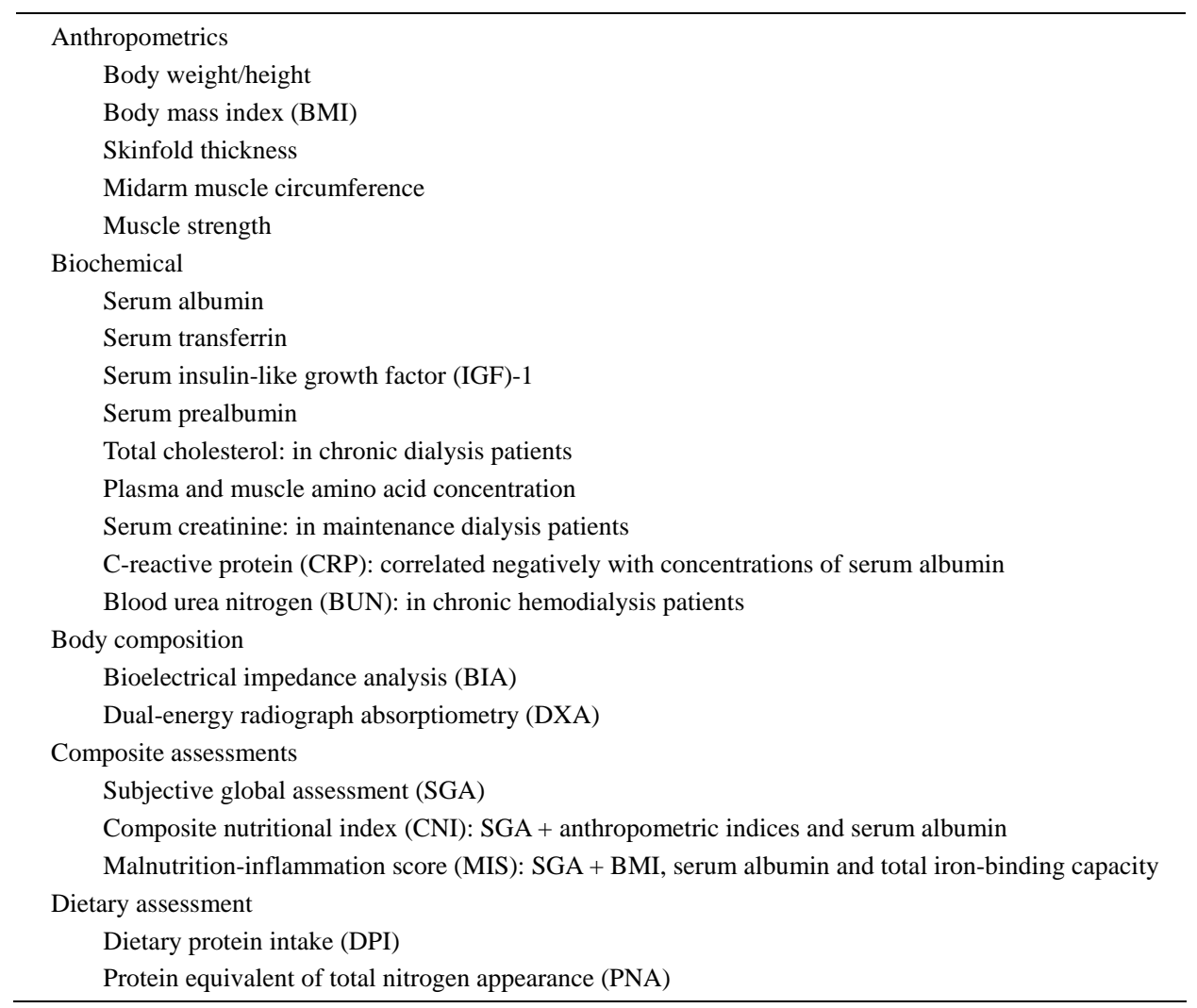


to measure lean body mass against TBN, which is considered to be a gold standard technique. These techniques are also influenced by ESRD-related limitations [63].

Subjective global assessment (SGA) has been also introduced for an overall clinical evaluation, including assessment of weight and weight change, dietary intake, gastrointestinal symptoms, and functional status. SGA has been relatively well correlated with objective measures of nutritional status in CKD and ESRD patients $[64,65]$. However, SGA is not yet a reliable predictor of degrees of uremic malnutrition. Furthermore, to overcome the lack of objectivity, standardization of guidelines and experience are very important for SGA [64].

\section{TREATMENT AND PREVENTION OF CHRONIC KIDNEY DISEASE-RELATED MALNUTRITION}

Since uremic malnutrition constitutes the most important factor for poor clinical outcomes in ESRD patients, novel strategies for treatment or prevention must be worked out (Table 2). Considering the complex and multifactorial pathogenesis of uremic malnutrition, the establishment of opinions about the treatment and prevention is not easy.

Inadequate nutritional intake and superimposed medical conditions are thought to play major roles in malnutrition and protein energy wasting, although uremia per se and its treatment modalities can also impair protein metabolism. The amount and/or route of protein and

Table 2. Practical recommendations for preventing or correcting malnutrition in chronic kidney disease.

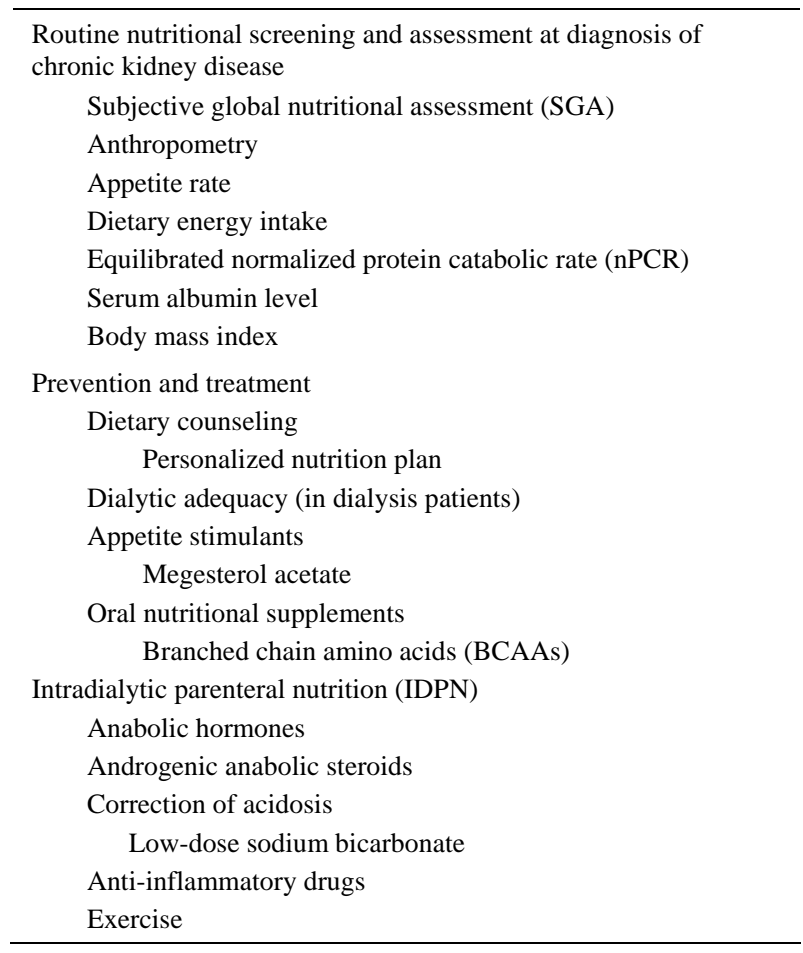

energy intake should be considered based on renal functional status; unlike predialysis CKD patients, ESRD patients on dialysis are encouraged to maintain an adequate protein and energy intake. Therefore, frequent comprehensive dietary counseling by nephrologists and dietitians is important.

Correction of metabolic acidosis in CKD patients can improve nutritional-related complications, such as nitrogen balance [66,67]. Therapy of uremic acidosis should aim for maintaining a serum bicarbonate level as close to normal as possible, for example 22 - $26 \mathrm{mmol} / \mathrm{L}$. The best way to initiate therapy is with oral sodium bicarbonate ( 1 tablet 3 times a day) and to increase the dosage as necessary [4]. The usual tablet of $650 \mathrm{mg}$ of sodium bicarbonate contains $7.5 \mathrm{mmol}$ of alkali. For patients who experience gastric discomfort with sodium bicarbonate, Shohl's solution, a mixture of sodium citrate and citric acid, is useful. In ESRD patients maintained on dialysis, the addition of alkali from the dialysate either as bicarbonate in HD or as lactate in PD may be used [44]. Since endogenous acid production, which depends on diet, is an important factor associated with uremic acidosis, ingestion of vegetables and fruits results in a net production of alkali which tends to delay the progression of uremic acidosis. However, development of hyperkalemia resulting from ingestion of foods containing high potassium content is a concern. Diuretic therapy and subsequent hypokalemia may also delay the development of acidosis, as these tend to stimulate ammonia production.

Once the early signs of uremic malnutrition are detected, active supplementation by an enteral or parenteral route should be considered. Despite conflicting results of oral nutritional supplementation in dialysis patients, oral protein, amino acid tablets or energy supplementation can be tried. According to several reports, oral amino acid supplementation significantly improved serum albumin concentration in HD patients and oral nutritional supplementation improved several nutritional parameters, including serum albumin and serum pre-albumin concentrations, in malnourished HD patients [68,69]. As an adjunctive nutritional therapy in CKD and ESRD patients, appetite stimulants such as megesterol acetate may be also considered, although the appropriate dose and side effect profiles remain to be evaluated in this drug [70]. Novel strategies such as anabolic hormones and anti-inflammatory drugs, along with conventional oral nutritional supplementation, may provide support for multiple patient populations. Recombinant human growth hormone (rhGH) administration has been suggested and has led to significant improvement in nitrogen balance, serum albumin, serum transferrin and IGF-1 concentrations [71]. Recombinant human IGF-1 (rhIGF-1) has also proposed as an anabolic agent, but the side effects of this agent are of concern in CKD patients [72]. As was men- 
tioned above, chronic inflammation is an important catabolic factor and new interventions for blocking the adverse effects of inflammation have been proposed. Since anti-inflammatory drugs such as thalidomide and COX-2 inhibitors would be theoretically helpful through inhibitory actions on TNF- $\alpha$ production and COX-2 expression, such strategies need to be evaluated in the future [51].

In dialysis patients, an adequate nutritional program can lead to improvement in nutritional status only when an optimal dialytic dose has been established, the catabolic stimulus possibly present has been counteracted, and drugs and procedures that reduce appetite have been avoided [73]. However, increasing the dose or using high-flux dialysis or hemodiafiltration with online regeneration of the ultrafiltrate does not seem to improve nutritional status.

If enteral nutritional supplementation is neither available nor effective, intradialytic parenteral nutrition (IDPN) is recommended for malnourished dialysis patients. Unlike early studies showing conflicting results about the beneficial effects of IDPN, IDPN has recently been reported to promote a robust increase in whole body proteolysis and a significant increase in forearm muscle protein synthesis in chronic HD patients [74]. In malnourished PD patients, conflicting results using amino acid dialysate as a nutritional intervention have been reported. Increases in serum transferrin and total protein concentrations as benefits from amino acid dialysate have been reported [75]. Because an increase in blood urea nitrogen concentration associated with exacerbation of uremic symptoms and metabolic acidosis represents a complication of amino acid dialysate use, these interventions should be considered only in PD patients with severe malnutrition. The use of both IDPN and AAD in ESRD patients need more study to evaluate any longterm beneficial effects. Furthermore, there are no data to show that active nutritional supplementation through the gastrointestinal tract is inferior to parenteral supplementation in dialysis populations [51]. The evidence from large-scale, well-designed nutritional intervention studies in CKD and ESRD patients with uremic malnutrition are very much needed.

Physical activity, as well as total daily protein intake, is the strongest predictor of the amount of lean body mass. Despite conflicting results, exercise appears to bring significant improvements in muscle attenuation, muscle strength, mid-thigh and mid-arm circumference, body weight, and CRP in patients on renal replacement therapy relative to non-exercising patients [76]. Longer training duration or more sensitive analytic techniques are required before such exercise regimens can be recommended as therapy for uremic malnutrition.

\section{CONCLUSIONS}

Since uremic malnutrition in patients with CKD, nutritional status progressively deteriorates as renal function worsens. The malnutrition in this population is associated with increased morbidity and mortality rates, as well as with numerous pre-existing factors. Therefore, it is vital to identify, treat and prevent conditions associated with poor clinical outcomes.

Despite the better understanding of the pathophysiologic mechanisms of uremic malnutrition and the improvements made in nutritional support, the nutritional condition of CKD and ESRD patients remains a significant cause for concern. Multimodal therapeutic strategies should be considered when the first signs of malnutrition are observed. More importantly, it is necessary to search actively for renal disease because early diagnosis and treatment can improve the prognosis for CKD patients and reduce economic costs connected with treatment.

\section{ACKNOWLEDGEMENTS}

This research was supported by Basic Science Research Program through the National Research Foundation of Korea (NRF) funded by the Minister of Education, Science and Technology (to C.W. Park; A111055).

\section{REFERENCES}

[1] Snyder, S. and Pendergraph, B. (2005) Detection and evaluation of chronic kidney disease. American Family Physician, 72, 1723-1732.

[2] Stevens, P.E., O’Donoghue, D.J., De Lusignan, S., et al. (2007) Chronic kidney disease management in the United Kingdom: NEOERICA project results. Kidney International, 72, 92-99. doi:10.1038/sj.ki.5002273

[3] Chin, H.J. and Kim, S. (2009) Chronic kidney disease in Korea. The Korean Journal Medine, 76, 511-514.

[4] Cibulka, R. and Racek, J. (2007) Metabolic disorders in patients with chronic kidney failure. Physiological Research, 56, 697-705.

[5] Levey, A.S., Eckardt, K.U., Tsukamoto, Y., et al. (2005) Definition and classification of chronic kidney disease: A position statement from kidney disease: Improving global outcomes (KDIGO). Kidney International, 67, 2089-2100. doi:10.1111/j.1523-1755.2005.00365.x

[6] Zhang, Q.L. and Rothenbacher, D. (2008) Prevalence of chronic kidney disease in population-based studies: Systematic review. BMC Public Health, 8, 117. doi:10.1186/1471-2458-8-117

[7] Pupim, L.B., Cuppari, L. and Ikizler, T.A. (2006) Nutrition and metabolism in kidney disease. Seminars in $\mathrm{Ne}$ phrology, 26, 134-157. doi:10.1016/j.semnephrol.2005.09.010

[8] Stenvinkel, P., Heimbürger, O., Paultre, F., et al. (1999) Strong association between malnutrition, inflammation, 
and atherosclerosis in chronic renal failure. Kidney International, 55, 1899-1911. doi:10.1046/j.1523-1755.1999.00422.x

[9] Savica, V., Santoro, D., Ciolino, F., et al. (2005) Nutritional therapy in chronic kidney disease. Nutrition in Clinical Care, 8, 70-76.

[10] Krebs, H.A. (1963) Renal gluconeogenesis. Advances in Enzyme Regulation, 1, 385-400. doi:10.1016/0065-2571(63)90034-7

[11] Cersosimo, E., Garlick, P. and Ferretti J. (1999) Insulin regulation of renal glucose metabolism in humans. American Journal of Physiology, 276, E78-E84.

[12] Cersosimo, E., Garlick, P. and Ferretti J. (1999) Renal glucose production during insulin-induced hypoglycemia in humans. Diabetes, 48, 261-266. doi:10.2337/diabetes.48.2.261

[13] Cersosimo, E., Garlick P. and Ferretti J. (2000) Renal substrate metabolism and gluconeogenesis during hypoglycemia in humans. Diabetes, 49, 1186-1193. doi:10.2337/diabetes.49.7.1186

[14] Meyer, C., Stumvoll, M., Dostou, J., et al. (2002) Renal substrate exchange and gluconeogenesis in normal postabsorptive humans. American Journal of Physiology, Endocrinology and Metabolism, 282, E428-E434.

[15] Fouque, D., Kalantar-Zadeh, K., Kopple, J., et al. (2008) A proposed nomenclature and diagnostic criteria for protein-energy wasting in acute and chronic kidney disease. Kidney International, 73, 391-398. doi:10.1038/sj.ki.5002585

[16] Fleet, M., Osman, F., Komaragiri, R., et al. (2008) Protein catabolism in advanced renal disease: Role of cytokines. Clinical Nephrology, 70, 91-100.

[17] Ikizler, T.A. (2004) Protein and energy: Recommended intake and nutrient supplementation in chronic dialysis patients. Seminars in Dialysis, 17, 471-478. doi:10.1111/j.0894-0959.2004.17608.x

[18] Kovacic, V., Roguljic, L. and Kovacic, V. (2003) Metabolic acidosis of chronically hemodialyzed patients. American Journal of Nephrology, 23, 158-164. doi:10.1159/000070205

[19] Mehrotra, R., Kopple, J.D. and Wolfson, M. (2003) Metabolic acidosis in maintenance dialysis patients: Clinical considerations. Kidney International Supplement, 88, S13-S25. doi:10.1046/j.1523-1755.2003.08802.x

[20] Lee, P.D., Conover, C.A. and Powell, D.R. (1993) Regulation and function of insulin-like growth factor-binding protein-1. Proceedings of the Society for Experimental Biology and Medicine, 204, 4-29.

[21] Alvestrand, A. (1997) Carbohydrate and insulin metabolism in renal failure. Kidney International Supplement, 62, S48-S52.

[22] Rigalleau, V. and Gin, H. (2005) Carbohydrate metabolism in uraemia. Current Opinion in Clinical Nutrition and Metabolic Care, 8, 463-469. doi:10.1097/01.mco.0000172590.32564.b9

[23] Ikizler, T.A. (2008) Nutrition, inflammation and chronic kidney disease. Current Opinion in Nephrology and Hy- pertension, 17, 162-167. doi:10.1097/MNH.0b013e3282f5dbce

[24] Attman, P.O., Samuelsson, O. and Alaupovic, P. (1993) Lipoprotein metabolism and renal failure. American Journal of Kidney Diseases, 21, 573-592.

[25] Guarnieri, G., Fonda, M., Situlin, R., et al. (1992) Effects of L-carnitine supplementation in the dialysate on serum lipoprotein composition of hemodialysis patients. Contributions to Nephrology, 98, 36-43.

[26] Kalantar-Zadeh, K., Ikizler, T.A., Block, G., et al. (2003) Malnutrition-inflammation complex syndrome in dialysis patients: Causes and consequences. American Journal of Kidney Diseases, 42, 864-881. doi:10.1016/j.ajkd.2003.07.016

[27] Kalantar-Zadeh, K., Stenvinkel, P., Bross, R., et al. (2005) Kidney insufficiency and nutrient-based modulation of inflammation. Current Opinion in Clinical Nutrition and Metabolic Care, 8, 388-396. doi:10.1097/01.mco.0000172578.56396.9e

[28] Garibotto, G., Sofia, A., Balbi, M., et al. (2007) Kidney and splanchnic handling of interleukin-6 in humans. $C y$ tokine, 37, 51-54. doi:10.1016/j.cyto.2007.02.015

[29] Tsujinaka, T., Fujita, J., Ebisui, C., et al. (1996) Interleukin 6 receptor antibody inhibits muscle atrophy and modulates proteolytic systems in interleukin 6 transgenic mice. The Journal of Clinical Investigation, 97, 244-249. doi:10.1172/JCI118398

[30] Könner, A.C., Klöckener, T. and Brüning, J.C. (2009) Control of energy homeostasis by insulin and leptin: Targeting the arcuate nucleus and beyond. Physiology \& Behavior, 97, 632-638. doi:10.1016/j.physbeh.2009.03.027

[31] Garibotto, G., Russo, R., Franceschini, R., et al. (1998) Inter-organ leptin exchange in humans. Biochemical and Biophysical Research Communications, 247, 504-509. doi:10.1006/bbrc.1998.8819

[32] Kalantar-Zadeh, K., Stenvinkel, P., Pillon, L., et al. (2003) Inflammation and nutrition in renal insufficiency. $A d$ vances in Renal Replacement Therapy, 10, 155-169. doi:10.1053/j.arrt.2003.08.008

[33] Franch, H.A. and Mitch, W.E. (2009) Navigating between the Scylla and Charybdis of prescribing dietary protein for chronic kidney diseases. Annual Review of Nutrition, 29, 341-64. doi:10.1146/annurev-nutr-080508-141051

[34] Galli, F. (2007) Protein damage and inflammation in uraemia and dialysis patients. Nephrology Dialysis Transplantation, 22, v20-v36. doi:10.1093/ndt/gfm294

[35] Roubenoff, R., Roubenoff, R.A., Cannon, J.G., et al. (1994) Rheumatoid cachexia: Cytokine-driven hypermetabolism accompanying reduced body cell mass in chronic inflammation. The Journal of Clinical Investigation, 93, 2379-2386. doi:10.1172/JCI117244

[36] Neyra, R., Chen, K.Y., Sun, M., et al. (2003) Increased resting energy expenditure in patients with end-stage renal disease. JPEN Journal of Parenteral and Enteral Nutrition, 27, 36-42. doi:10.1177/014860710302700136

[37] Borah, M.F., Schoenfeld, P.Y., Gotch, F.A., et al. (1978) 
Nitrogen balance during intermittent dialysis therapy of uremia. Kidney International, 14, 491-500. doi:10.1038/ki.1978.154

[38] Klaunig, J.E., Xu, Y., Isenberg, J.S., et al. (1998) The role of oxidative stress in chemical carcinogenesis. Environmental Health Perspectives, 106, 289-295.

[39] Lim, P.S., Wei, Y.H., Yu, Y.L., et al. (1999) Enhanced oxidative stress in haemodialysis patients receiving intravenous iron therapy. Nephrology Dialysis Transplantation, 14, 2680-2687. doi:10.1093/ndt/14.11.2680

[40] Chan, W., Valerie, K.C. and Chan, J.C. (1993) Expression of insulin-like growth factor-1 in uremic rats: Growth hormone resistance and nutritional intake. Kidney International, 43, 790-795. doi:10.1038/ki.1993.112

[41] Schaefer, F., Chen, Y., Tsao, T., et al. (2001) Impaired JAK-STAT signal transduction contributes to growth hormone resistance in chronic uremia. The Journal of Clinical Investigation, 108, 467-475.

[42] Brüngger, M., Hulter, H.N. and Krapf, R. (1997) Effect of chronic metabolic acidosis on the growth hormone/ IGF-1 endocrine axis: New cause of growth hormone insensitivity in humans. Kidney International, 51, 216-221. doi:10.1038/ki.1997.26

[43] Kraut, J.A. and Kurtz, I. (2005) Metabolic acidosis of CKD: Diagnosis, clinical characteristics, and treatment. American Journal of Kidney Diseases, 45, 978-993. doi:10.1053/j.ajkd.2005.03.003

[44] Oh, M.S., Uribarri, J., Weinstein, J., et al. (2004) What unique acid-base considerations exist in dialysis patients? Seminars in Dialysis, 17, 351-364. doi:10.1111/j.0894-0959.2004.17342.x

[45] Garibotto, G., Russo, R., Sofia, A., et al. (1994) Skeletal muscle protein synthesis and degradation in patients with chronic renal failure. Kidney International, 45, 14321439. doi:10.1038/ki.1994.187

[46] Pollock, C.A., Ibels, L.S., Zhu, F.Y., et al. (1997) Protein intake in renal disease. Journal of the American Society of Nephrology, 8, 777-783. doi:10.1038/ki.1994.187

[47] Pupim, L.B., Kent, P., Caglar, K., et al. (2002) Improvement in nutritional parameters after initiation of chronic hemodialysis. American Journal of Kidney Diseases, 40, 143-151. doi:10.1053/ajkd.2002.33923

[48] Bossola, M., Muscaritoli, M., Tazza, L., et al. (2005) Malnutrition in hemodialysis patients: What therapy? American Journal of Kidney Diseases, 46, 371-386. doi:10.1053/j.ajkd.2005.05.031

[49] Ikizler, T.A., Pupim, L.B., Brouillette, J.R., et al. (2002) Hemodialysis stimulates muscle and whole body protein loss and alters substrate oxidation. American Journal of Physiology, Endocrinology and Metabolism, 282, E107E116.

[50] Parker, T.F. III., Wingard, R.L., Husni, L., et al. (1996) Effect of the membrane biocompatibility on nutritional parameters in chronic hemodialysis patients. Kidney International, 49, 551-556. doi:10.1038/ki.1996.78

[51] Pupim, L.B. and Ikizler, T.A. (2003) Uremic malnutrition: New insights into an old problem. Seminars in Dialysis, 16, 224-232. doi:10.1046/j.1525-139X.2003.16046.X
[52] Ikizler, T.A., Wingard, R.L., Sun, M., et al. (1996) Increased energy expenditure in hemodialysis patients. Journal of the American Society of Nephrology, 7, 26462653.

[53] Kaysen, G.A. and Levin, N.W. (2002) Why measure serum albumin levels? Journal of Renal Nutrition, 12, 148-150. doi:10.1053/jren.2002.33509

[54] Kaysen, G.A., Dubin, J.A., Müller, H.G., et al. (2002) Relationships among inflammation nutrition and physicologic mechanisms establishing albumin levels in hemodialysis patients. Kidney International, 61, 2240-2249. doi:10.1046/j.1523-1755.2002.00076.x

[55] Kaysen, G.A., Stevenson, F.T. and Depner, T.A. (1997) Determinants of albumin concentration in hemodialysis patients. American Journal of Kidney Diseases, 29, 658668. doi:10.1016/S0272-6386(97)90117-7

[56] Kaysen, G.A., Rathore, V., Shearer, G.C., et al. (1995) Mechanisms of hypoalbuminemia in hemodialysis patients. Kidney International, 48, 510-516. doi:10.1038/ki.1995.321

[57] Neyra, N.R., Hakim, R.M., Shyr, Y., et al. (2000) Serum transferrin and serum prealbumin are early predictors of serum albumin in chronic hemodialysis patients. Journal of Renal Nutrition, 10, 184-190. doi:10.1053/jren.2000.16325

[58] Chertow, G.M., Ackert, K., Lew, N.L., et al. (2000) Prealbumin is as important as albumin in the nutritional assessment of hemodialysis patients. Kidney International, 58, 2512-2517. doi:10.1046/j.1523-1755.2000.00435.x

[59] Holland, D.C., Meers, C., Lawlor, M.E., et al. (2001) Serial prealbumin levels as predictors of outcomes in a retrospective cohort of peritoneal and hemodialysis patients. Journal of Renal Nutrition, 11, 129-138. doi:10.1053/jren.2001.24358

[60] Suliman, M.E., Lindholm, B., Bárány, P., et al. (2001) Hyperhomocysteinemia in chronic renal failure patients: Relation to nutritional status and cardiovascular disease. Clinical Chemistry and Laboratory Medicine, 39, 734738. doi:10.1515/CCLM.2001.122

[61] Perna, A.F., Ingrosso, D., Satta, E., et al. (2004) Homocysteine metabolism in renal failure. Current Opinion in Clinical Nnutrition and Metabolic Care, 7, 53-57. doi:10.1097/00075197-200401000-00010

[62] Kerr, P.G., Strauss, B.J. and Atkins, R.C. (1996) Assessment of the nutritional state of dialysis patients. Blood Purification, 14, 382-387. doi:10.1159/000170290

[63] Chertow, G.M., Lazarus, J.M., Lew, N.L., et al. (1997) Bioimpedance norms for the hemodialysis population. Kidney International, 52, 1617-1621. doi:10.1038/ki.1997.493

[64] Detsky, A.S., McLaughlin, J.R., Baker, J.P., et al. (1987) What is subjective global assessment of nutritional status? JPEN Journal of Parenteral and Enteral Nutrition, 11, 813. doi:10.1177/014860718701100108

[65] Cooper, B.A., Bartlett, L.H., Aslani, A., et al. (2002) Validity of subjective global assessment as a nutritional marker in end-stage renal disease. American Journal of Kidney Diseases, 40, 126-132. 
doi:10.1053/ajkd.2002.33921

[66] Papadoyannakis, N.J., Stefanidis, C.J. and McGeown, M. (1984) The effect of the correction of metabolic acidosis on nitrogen and potassium balance of patients with chronic renal failure. The American Journal of Clinical Nutrition, 40, 623-627.

[67] Price, S.R. and Mitch, W.E. (1994) Metabolic acidosis and uremic toxicity: Protein and amino acid metabolism. Seminars in Nephrology, 14, 232-237.

[68] Eustace, J.A., Coresh, J., Kutchey, C., et al. (2000) Randomized double-blind trial of oral essential amino acids for dialysis-associated hypoalbuminemia. Kidney International, 57, 2527-2538. doi:10.1046/j.1523-1755.2000.00112.x

[69] Caglar, K., Fedje, L., Dimmitt, R., et al. (2002) Therapeutic effects of oral nutritional supplementation during hemodialysis. Kidney International, 62, 1054-1059. doi:10.1046/j.1523-1755.2002.00530.x

[70] Burrowes, J.D., Bluestone, P.A., Wang, J., et al. (1999) The effects of moderate doses of megestrol acetate on nutritional status and body composition in a hemodialysis patient. Journal of Renal Nutrition, 9, 89-94. doi:10.1016/S1051-2276(99)90006-7

[71] Kotzmann, H., Yilmaz, N., Lercher, P., et al. (2001) Dif- ferential effects of growth hormone therapy in malnourished hemodialysis patients. Kidney International, 60, 1578-1585. doi:10.1046/j.1523-1755.2001.00971.x

[72] Hammerman, M.R. (2000) Insulin-like growth factor I treatment for end-stage renal disease at the end of the millennium. Current Opinion in Nephrology and Hypertension, 9, 1-3. doi:10.1097/00041552-200001000-00001

[73] Bossola, M., Muscaritoli, M., Tazza, L., et al. (2005) Malnutrition in hemodialysis patients: What therapy? American Journal of Kidney Diseases, 46, 371-386. doi:10.1053/j.ajkd.2005.05.031

[74] Pupim, L.B., Flakoll, P.J., Brouillette, J.R., et al. (2002) Intradialytic parenteral nutrition improves protein and energy homeostasis in chronic hemodialysis patients. The Journal of Clinical Investigation, 110, 483-492.

[75] Jones, M., Hagen, T., Boyle, C.A., et al. (1998) Treatment of malnutrition with $1.1 \%$ amino acid peritoneal dialysis solution: Results of a multicenter outpatient study. American Journal of Kidney Diseases, 32, 761-769. doi:10.1016/S0272-6386(98)70131-3

[76] Mak, R.H. and Cheung, W. (2007) Therapeutic strategy for cachexia in chronic kidney disease. Current Opinion in Nephrology and Hypertension, 16, 542-546. doi:10.1097/MNH.0b013e3282f02204 